\title{
Gestão de dermatite associada à incontinência pelo enfermeiro: revisão integrativa
}

RESUMO | Objetivo:descrever o gerenciamento da dermatite associada a incontinência fecal e urinária pelo profissional enfermeiro. Método:Trata-se de um estudo bibliográfico, tipo revisão integrativa. Realizou-se a busca por artigos; publicados no período de 2015 a 2019; nos idiomas português, inglês e espanhol; disponíveis na íntegra. Foram consultadas as seguintes plataformas de dados: BIREME, LILACS e SciELO. Os dados foram organizados e apresentados em figuras e tabelas. Resultado: Dos 501 estudos encontrados, 32 foram selecionados para a leitura na integra; contudo, após a leitura permaneceram apenas os que atendiam aos critérios descritos na metodologia, totalizando 22 estudos. Após a leitura dos estudos selecionados, os artigos foram categorizados em recortes temáticos, classificando o conhecimento produzido acerca do tema.Conclusão:De uma forma geral, todos os estudos apontaram para a importância de uma adequada inspeção da pele, constituindo-se como o "gold standard" que quer dizer "Padrão ouro" da prevenção e diagnóstico de DAl.

Palavras-chaves: Dermatite, Profissionais de Enfermagem, Lesão por Pressão, Incontinência Urinaria.

ABSTRACT | Objective: to describe the management of dermatitis associated with fecal and urinary incontinence by the nurse professional. Method: This is a bibliographic study, type integrative review. The search for articles was carried out; published in the period from 2015 to 2019; in Portuguese, English and Spanish; available in full. The following data platforms were consulted: BIREME, LILACS and SciELO. The data were organized and presented in figures and tables. Result: Of the 501 studies found, 32 were selected for full reading; however, after reading, only those who met the criteria described in the methodology remained, totaling 22 studies. After reading the selected studies, the articles were categorized into thematic clippings, classifying the knowledge produced on the topic. Conclusion: In general, all studies pointed out the importance of an adequate skin inspection, constituting itself as the "gold standard", which means "gold standard" for the prevention and diagnosis of AID.

Keywords: Dermatitis,Nurse Practitioners, PressureUlcer, Urinarylncontinence.

RESUMEN | Objetivo: describirel manejo de ladermatitisasociada a incontinencia fecal y urinaria por parte delprofesional de enfermería. Método: Se trata de unestudio bibliográfico, tipo revisión integradora. Se realizólabúsqueda de artículos; publicado enel período de 2015 a 2019; enportugués, inglés y español; disponibleensutotalidad. Se consultaronlassiguientes plataformas de datos:BIREME, LILACS y SCiELO. Los datosfueron organizados y presentados en figuras y tablas. Resultado: de los 501 estudios encontrados, 32 fueronseleccionados para lectura completa; sin embargo, luego de lalectura, solo quedaronaquellos que cumplieron com Los criterios descritosenlametodología, totalizando 22estudios. Luego de lalectura de losestudiosseleccionados, los artículos fueron categorizados en recortes temáticos, clasificandoelconocimientoproducido sobre el tema. Conclusión: En general, todos losestudiosapuntan a laimportancia de una adecuadainspeccióncutánea, constituyéndose como el "estándar de oro", que significa "estándar de oro" para laprevención y diagnóstico de la SIDA.

Palavras claves: Dermatitis, EnfermerasPracticantes, Úlcera por Presión, IncontinenciaUrin.

\section{Joel Azevedo de Menezes Neto}

Pós-Graduando em Enfermagem em Estomaterapia - Estomias, Feridas e Incontinência pelo Albert Einstein Instituto Israelita de Ensino e Pesquisa. Docente na Faculdade do Belo Jardim (FBJ). Belo Jardim, Pernambuco (PE), Brasil.

ORCID: 0000-0002-9467-4975

\section{Elaine Batista de Melo}

Pós-graduanda em Enfermagem em Urgência e Emergência e Unidade de Terapia Intensiva (UTI) pelo Grupo CEFAPP (Centro de Formação, Pós-graduação e Pesquisa em Saúde). Recife, Pernambuco $(\mathrm{PE})$, Brasil.

ORCID: 0000-0002-6313-4837

Alexandra Waleska de Oliveira Aguiar

Mestre em Enfermagem pelo Programa Associado de Pós-graduação da Universidade de Pernambuco/Universidade Estadual da Paraíba (UPE/UEPB). Coordenadora do Curso de Graduação em Enfermagem da Faculdade do Belo Jardim (FBJ). Belo Jardim, Pernambuco (PE), Brasil. ORCID: 0000-0001-5552-2410

Franciskellyde Siqueira Pessôa
Doutoranda em Saúde Pública e Mestra
em Saúde Coletiva pela Faculdade
Interamericana de Saúde (FICS).
Coordenadora do Curso de Graduação
em Enfermagem no Centro Universitário
Maurício de Nassau (UNINASSAU).
Caruaru, Pernambuco (PE), Brasil.
ORCID: 0000-0002-4073-9120

\section{Marcos Antonio de Oliveira Souza}

Doutor e Mestre em Enfermagem pelo Programa Associado de Pós-graduação da Universidade de Pernambuco/ Universidade Estadual da Paraíba (UPE/ UEPB). Especialista em Estomaterapia pela UPE. Recife, Pernambuco (PE), Brasil. ORCID: 0000-0002-9087-5788

\section{Liniker Scolfild Rodrigues da Silva}

Especialista em Saúde Coletiva na modalidade Residência pela Faculdade de Ciências Médicas (FCM)/Universidade de Pernambuco (UPE). Especialista em Enfermagem Obstétrica na modalidade Residência pela Faculdade de Enfermagem Nossa Senhora das Graças (FENSG)/UPE. Recife, Pernambuco (PE), Brasil. ORCID: 0000-0003-3710-851X 


\section{INTRODUÇÃO}

T endo em vista que a pele é o maior órgão do corpo humano, estudosa definem como um envoltório com função de revestimento e proteção a órgãos mais complexos, isolando o meio interno do externo, ainda funcional e sofisticada segundo os autores, por suas renovaçõese reparações a todo o momento. Ocupa lugar de importância na esfera psíquica do ser humano, sendo a responsável pela ligação do indivíduo ao meio externo ${ }^{1,2}$.

Vários fatorem podem gerar alterações na pele, dentre eles, a umidade. $\mathrm{O}$ dano à pele associado à umidade (Moisture Associated SkinDamage - MASD) é retratado como inflamação e erosão cutânea provocadapela exposição prolongada da pele à urina, fezes, suor, exsudato de feridas e muco. Relacionados a estas exposições, ainda deve-se considerar outros fatores etiológicos como a ocorrência de hiperhidratação, presença de fricção e temperatura da pele. As quatro formas de MASD são a Dermatite Intertriginosa, Dermatite Peristomal, Dermatite Periférica e a Dermatite Associada à Incontinência (DAl) ${ }^{2,3}$.

Destaca-se a DAI, que surge normalmente em pacientes com incontinência urinária e/ou fecal. Trata-se de uma condição bastante frequente, tendo em conta que existe um número significativode pessoas, maioritariamente com 65 e mais anos de idade, internadas em cuidados agudos ou de longa duração, que sofrem de incontinência urinária e/ou fecal².

O contato por um período extenso com urina e fezes eleva a incidência desse tipo de lesão, caracterizada por inflamação da pele com aspecto inicial de hiperemia, podendo evoluir para ruptura da pele, denominada ulceração. Tendo como principal fator de risco, a presença de incontinência.A exposição prolongada a um meio continuamente úmido gerano local uma maceração cutânea que ao evoluir para erosão da epiderme, deixa a pele mais propícia ao efeito prejudicial da pressão, elevando a disposição para o surgimento de úlceras por pressão, para infeção por Staplylococcus, podendo evoluir anda para celulite e fasceíte necrosante ${ }^{2-4}$.

Esse agravo tem um efeito considerável sobre o bem-estar físico e psicológico das pessoas levando-as a apresentar desconforto, dor, queimação, prurido, formigamento nas áreas afetadas, excessivo incômodo durante os cuidados, transtorno na execução de atividades de vida diária, no sono com consequente redução na qualidade de vida ${ }^{3}$.

Uma vez que, a pele é uma variável sensível aos cuidados de enfermagem, a estrutura e o processo pelo qual os cuidados de enfermagem são fornecidosacabam influenciandoseus resultados, necessitando assim, de evidência científica disponível, visando preencher os vazios na prestação de cuidados e na identificação de fatores de risco. Precisa-se imprescindivelmentedo desenvolvimento de uma estratégia de prevenção, tratamento e gestão para que haja melhores cuidados e desse modo, melhor qualidade de vida ${ }^{3-5}$.

A prevalência da DAl é pouco conhecida, e os dados internacionais de prevalência de Incontinência mostram valores que vão de 1,9 a $21,3 \%$ da população, elevando-se com o aumento da faixa etária5. Desta forma, percebe-se a importância e relevância deste trabalho para empoderar as pesquisas científicas e aprimorar os conhecimentos dos profissionais de enfermagem sobre a DAl e seus aspectos para detecção, manejo adequado e prevenção. Tais colocações são de grande importância para subsidiar e auxiliar no traçar de uma assistência à saú- de de forma ampla e eficaz.

O presente estudo após todo descrito e buscando responder a seguinte questão norteadora da pesquisa: "Como é realizado o gerenciamento da dermatite associada a incontinência fecal e urinária pelo profissional enfermeiro?", tem como objetivo, descrever o gerenciamento da dermatite associada a incontinência fecal e urinária pelo profissional enfermeiro.

\section{MÉTODO}

Trata-se de um estudo bibliográfico, tipo revisão integrativa onde realizou-se a busca de artigos para desenvolvimento metodológico e foram percorridas, as seguintes etapas: elaboração da questão norteadora e objetivo do estudo; definição de critérios de inclusão e exclusão das produções científicas; busca de estudos científicos nas bases de dados e bibliotecas virtuais; análise e categorização das produções encontradas; resultados e discussão dos achados6.

Para o levantamento da questão norteadora, aplicou-se a estratégia PICO (P-população/paciente, I-intervenção/interesse, C-comparação/Ausência e O-desfecho) onde $\mathrm{C}$ permaneceu ausente, pois não houveram elementos de cunho comparativo. Estratégia baseada na segmentação da hipótese, que visa o levantamento de dados de forma sistematizada ${ }^{7}$.

Desta forma, definiu-se a seguinte questão norteadora da pesquisa: "Como é realizado o gerenciamento da dermatite associada a incontinência fecal e urinária pelo profissional enfermeiro?" (Figura 1).
Figura 1: Definição da questão norteadora de acordo com a estratégia de PICO. Recife, Pernambuco (PE), Brasil, 2020.

População/Problema - Pacientes com Dermatite Associada a Incontinência.

Intervenção/Interesse - Processo de Gerenciamento.

Comparação/Ausência -

Desfecho - Intervenções Adequadas.

Fonte: Elaboração própria. 
Para seleção dos artigos, utilizaram-se como critérios de inclusão: ser artigo original, ter sido publicado em português, inglês ou espanhol, no período de 2015 a 2019, e estar disponível na íntegra. Foram excluídos teses, dissertações e monografias, editoriais, estudos de caso, bem como estudos repetidos encontrados em mais de uma base de dados ou biblioteca virtual além de artigos que não responderam à questão norteadora.

O levantamento dos dadosocorreu no período de fevereiro a março de 2020 nas seguintes bases de dados e bibliotecas virtuais: na BIREME, na Literatura Latino-Americana e do Caribe em Ciências da Saúde (LILACS); na Scientific Electronic Library Online (SciELO) e revistas eletrônicas de enfermagem. Elegendo estas bases de dados e bibliotecas por entender que atingem a literatura publicada, como também referências técnico-científicas brasileiras em saúde pública e coletiva. Foram realizados cruzamentos dos descritores "Dermatite associada a Incontinência", "gerenciamento de DAI",
"Enfermagem" e "Feridas". presentes na base de Descritores em Ciências da Saúde (DecS) combinados com operador booleano OR, efetuando busca conjunta e individual para que possíveis diferenças fossem corrigidas.

A seleção dos estudos, baseou-se no PreferredReportingltems for SystematicReviewand Meta-Analyse - PRISMA um checklist com 27 itens e um fluxograma de quatro etapas, com o objetivo de auxiliar no desenvolvimento de $\operatorname{artigos}^{8}$. A princípio eliminaram-se por meio da leitura de títulos e resumos, estudos duplicados. Destes pré-selecionados, realizou-se leitura na íntegra, a fim de verificar os que atendem à questão norteadora e aos critérios de inclusão/exclusão. Construiu-se então a amostra final com estudos pertinentes aos critérios pré-estabelecidos (Figura 2).

O nível de evidência dos estudos selecionados foi determinado de acordo com a Agency for Healthcare Research and Quality (AHRQ, 2016) ${ }^{9}$ : Nível I- Metanálise de múltiplos estudos controlados;

Figura 2: Fluxograma da seleção dos estudos segundo o Preferred Reporting Items for Systematic Reviewsand Meta-Analyses (PRISMA 2015). Recife, Pernambuco (PE), Brasil, 2020.

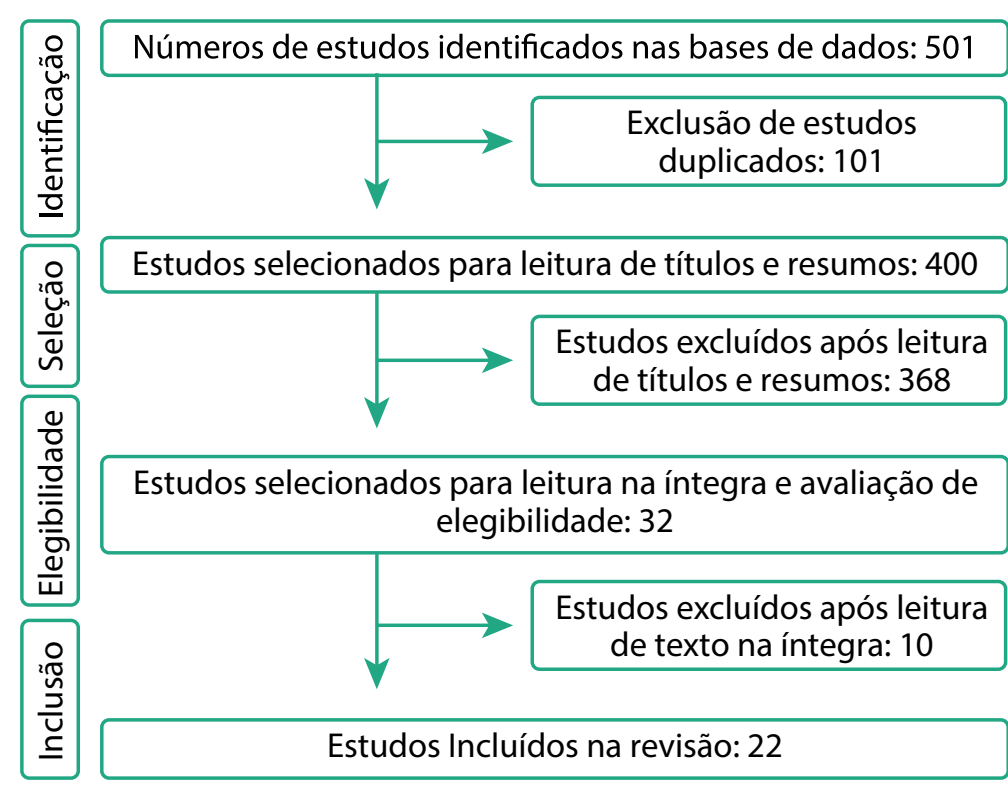

Fonte: Elaboração própria.
Nível II- Estudos individuais com delineamento experimental; Nível III- Estudo com delineamento quase-experimental como estudo sem randomização com grupo único pré e pós-teste, séries temporais ou caso-controle; Nível IV- Estudo com delineamento não-experimental como pesquisa descritiva correlacional e qualitativa ou estudos de caso; Nível V- Relatório de casos ou dado obtido de forma sistemática, de qualidade verificável ou dados de avaliação de programas; e por fim o Nível VI- Opiniões de autoridades respeitáveis baseada na competência clínica ou opinião de comitês de especialistas, incluindo interpretações de informações não baseadas em pesquisas.

Visando simplificar o entendimento das publicações selecionadas nesta revisão integrativa, organizaram-se os dados em figuras e quadro, expostos de forma descritiva.

\section{RESULTADOS}

Fazendo uma análise após leitura dos artigos foi constatado que no presente estudo a prevalência de incontinência urinária foi de 33,33\% dos idosos institucionalizados. No mínimo a metade dos idosos hospitalizados é acometida pela incontinência urinária, contribuindo para problemas físicos e psicossociais. Os fatores que prejudicam a pele perineal são aqueles como hidratação, umidade por longa permanência, fricção, $\mathrm{pH}$ da urina e/ou fezes e patógenos já presentes nas fezes ou na microbiota da pele.

As mulheres apresentam maior ocorrência de incontinência urinária do que os homens, sendo afetadas em todos os grupos etários. Evidenciamos que pacientes com maior porcentagem de incontinência urinária são mulheres $77,77 \%$ do que para os homens 22,22\%. Apesar dos sintomas leves, a DAI frequentemente produz desconforto $78 \%$ apresentam este sintoma e 38\% dos indivíduos apresentando também sintomas de prurido ou queimação.

Em um dos estudos foi constatado 
150 idosos institucionalizados, a incontinência urinária teve maior prevalência nas mulheres $62,6 \%$ do que em homens $45,7 \%$. O risco maior de incontinência urinária relacionada ao sexo feminino deve-se pelo fato de diferenças anatômicas como diferenças no comprimento uretral feminino que se apresenta maior nos homens; a anatomia do assoalho pélvico; além da diminuição da pressão de fechamento uretral, associados à hipermobilidade do colo vesical e ao enfraquecimento na musculatura do assoalho pélvico, devido aos efeitos da gestação e do parto no mecanismo de continência, além das alterações hormonais decorren- tes da menopausa.

Em relação aos homens que fazem tratamento de câncer prostático, os que passam por prostatectomia radical (PR) e também os que realizam braquiterapia (BT), apresentam incontinência urinária. $A$ incidência de incontinência urinaria foi maior nos pacientes que realizaram PR em comparação aos que realizaram tratamento por BT. Os dados dos que apresentaram IU como sequela destes tratamentos foram $66 \%$ dos homens que fizeram $P R$, e em 33\% dos que fizeram BT.

Em estudos realizados em unidades hospitalares foi evidenciado que $86 \%$ dos enfermeiros possuem conhecimen- to para manejar a DAl leve e os casos moderados ou severos e gerenciar este processo de complicação dermatológica e diferenciar de LPP estágio1, incluindo descamação da pele e bolhas, ficam sob a responsabilidade da comissão de lesões de pele/curativos da insti $\neg$ tuição, se houver pedido de avaliação por parte da equipe médica em hospital hospitalar ou do próprio enfermeiro gerente da comissão de curativos, por isso a equipe deve estar orientada a informar o enfermeiro aos sinais precoces da DAI. Todos os resultados respondem criteriosamente os objetivos e apresentam total aderência ao processo do trabalho.

Quadro 1: Distribuição dos artigos segundo sua caracterização quanto: autores, objetivo, tipo do estudo e ano de publicação. Recife, Pernambuco (PE), Brasil, 2020.

\begin{tabular}{|c|c|c|c|c|c|}
\hline Q & AUTORES & OBJETIVO & $\mathrm{N}^{*}$ & $\begin{array}{l}\text { TIPO DE } \\
\text { ESTUDO }\end{array}$ & $\begin{array}{c}\text { ANO DE } \\
\text { PUBLICAÇÃO }\end{array}$ \\
\hline 1 & $\begin{array}{c}\text { QuadrosLB, Aguiar A, Menezes AV, Alves EF, } \\
\text { Nery T, Bezerra PP. }\end{array}$ & $\begin{array}{l}\text { Determinar a prevalência de incontinência urinária } \\
\text { em uma amostra de idosos institucionalizados e } \\
\text { analisar sua relação com características socio- } \\
\text { demográficas, comorbidades associadas, função } \\
\text { cognitiva e independência funcional. }\end{array}$ & IV & $\begin{array}{l}\text { Estudo } \\
\text { transversal, } \\
\text { descritivo e } \\
\text { exploratório. }\end{array}$ & 2015 \\
\hline 3 & $\begin{array}{l}\text { Chianca TCM, Gonçales PR, Salgado PO, } \\
\text { Machado BO, Amorim GL, Alcoforado CLGC. }\end{array}$ & $\begin{array}{l}\text { Estimar incidência, determinar fatores de risco e } \\
\text { propor modelo de predição de risco para desen- } \\
\text { volvimento de dermatite associada a incontinência } \\
\text { em pacientes adultos críticos. }\end{array}$ & IV & $\begin{array}{l}\text { Estudo descri- } \\
\text { tivo. }\end{array}$ & 2016 \\
\hline 5 & $\begin{array}{c}\text { Tavares AP, Ramos AF, Vieira EV, Mendon- } \\
\text { çaS,Fonseca C, Lopes MJ, et al. }\end{array}$ & $\begin{array}{l}\text { Identificar quais as intervenções de enfermagem } \\
\text { dirigidas à prevenção e cicatrização da DAl, em } \\
\text { cuidados agudos e de longa duração. }\end{array}$ & V & $\begin{array}{l}\text { Revisão } \\
\text { integrativa. }\end{array}$ & 2017 \\
\hline 6 & $\begin{array}{c}\text { Alves LAF, Santana RF, Cardozo AS, Souza } \\
\text { TM, Silva CFR. }\end{array}$ & $\begin{array}{l}\text { Analisar o uso da fralda geriátrica ao que concerne } \\
\text { a técnica, os produtos e os eventos adversos e a } \\
\text { partir disso formular um protocolo de intervenção } \\
\text { para uso de fraldas geriátricas. }\end{array}$ & V & $\begin{array}{l}\text { Revisão siste- } \\
\text { mática. }\end{array}$ & 2017 \\
\hline
\end{tabular}




\begin{tabular}{|c|c|c|c|c|c|}
\hline 8 & $\begin{array}{c}\text { Nascimento DC, Cunha CV, PennaLHG,Sou- } \\
\text { zaNVDO,Marques GS. }\end{array}$ & $\begin{array}{l}\text { Analisar produções científicas que abordam esse } \\
\text { tipo de lesão considerando seus conceitos e } \\
\text { medidas de prevenção e tratamentos propostos. }\end{array}$ & $\mathrm{V}$ & $\begin{array}{l}\text { Revisão } \\
\text { integrativa. }\end{array}$ & 2016 \\
\hline 9 & Greco APC, Guimarães AF, NascimentoTS & $\begin{array}{l}\text { Descrever a utilização do equipamento de con- } \\
\text { trole de IA e demonstrar sua eficácia no manejo } \\
\text { de pacientes com DAI. }\end{array}$ & IV & $\begin{array}{l}\text { Qualitativo, } \\
\text { descritivo, } \\
\text { retrospectivo. }\end{array}$ & 2017 \\
\hline 10 & Ramos AF, Pinto MCPF & $\begin{array}{l}\text { Elaborar espelho demonstrativo de medidas pre- } \\
\text { ventivas através de literatura e folder explicativo } \\
\text { sobre medidas de prevenção de DAl. }\end{array}$ & $\mathrm{V}$ & $\begin{array}{l}\text { Revisão siste- } \\
\text { mática. }\end{array}$ & 2018 \\
\hline 11 & $\begin{array}{c}\text { SaudLF, Campos GV, ManiezzoR, Marques } \\
\text { YMP, Rezende LF }\end{array}$ & $\begin{array}{l}\text { Desenvolver uma revisão sistemática sobre a } \\
\text { incidência de incontinência urinária em homens } \\
\text { submetidos aos tratamentos de braquiterapia e } \\
\text { prostatectomia. }\end{array}$ & V & $\begin{array}{l}\text { Revisão } \\
\text { integrativa }\end{array}$ & 2018 \\
\hline 12 & $\begin{array}{l}\text { Alcoforado CLGC, Machado BO, Campos } \\
\text { CC, Gonçales PC,Ercole FF, Chianca TCM. }\end{array}$ & $\begin{array}{l}\text { Identificar, na literatura, as melhores evidências } \\
\text { sobre os fatores de risco para o desenvolvimen- } \\
\text { to da Dermatite Associada à Incontinência (DAI). }\end{array}$ & V & $\begin{array}{l}\text { Revisão } \\
\text { integrativa. }\end{array}$ & 2018 \\
\hline 13 & Silva RM, Fernandes FAV & $\begin{array}{c}\text { Mapear as competências específicas do Gestor } \\
\text { de Feridas. }\end{array}$ & V & $\begin{array}{l}\text { Revisão } \\
\text { sistemática de } \\
\text { escopo. }\end{array}$ & 2019 \\
\hline 14 & Rocha Filho JS, Carvalho CGN. & $\begin{array}{l}\text { Prover informações acerca de um subtipo morfo- } \\
\text { lógico raro de dermatite irritativa das fraldas na } \\
\text { prática clínica, a DJ. }\end{array}$ & $\mathrm{V}$ & $\begin{array}{l}\text { Revisão } \\
\text { integrativa. }\end{array}$ & 2017 \\
\hline 15 & $\begin{array}{l}\text { Alves AT, Jácomo RH, Epaminondas WA, } \\
\text { Faria DS, Leite RM, GomideLB,et al. }\end{array}$ & $\begin{array}{l}\text { Analisar a efetividade da intervenção educativa } \\
\text { na prevenção e tratamento da dermatite da } \\
\text { fralda de idosos institucionalizados. }\end{array}$ & IV & $\begin{array}{l}\text { Estudo } \\
\text { qualitativo, } \\
\text { longitudinal e } \\
\text { prospectivo }\end{array}$ & 2015 \\
\hline 16 & Bitencourt GR, Santana RF. & $\begin{array}{l}\text { Analisar instrumentos de avaliação dos eventos } \\
\text { adversos associados ao uso de fraldas geriátricas. }\end{array}$ & V & $\begin{array}{l}\text { Revisão } \\
\text { integrativa. }\end{array}$ & 2019 \\
\hline 17 & $\begin{array}{l}\text { Lage MA, Silva TBC, Evangelista DR, Rezen- } \\
\text { de FAC, Silva Netto LS, Osório NB, et al. }\end{array}$ & $\begin{array}{c}\text { Caracterizar a prevalência de incontinência urinária } \\
\text { e fecal nos idosos. }\end{array}$ & IV & $\begin{array}{l}\text { Estudo trans- } \\
\text { versal. }\end{array}$ & 2019 \\
\hline 18 & Leme LNR, Souza NVDO, Chagas PF. & $\begin{array}{l}\text { Revisar a produção científica sobre incontinência } \\
\text { anal (IA), atualizando o conhecimento de enferma- } \\
\text { gem sobre o tema. }\end{array}$ & V & $\begin{array}{l}\text { Revisão } \\
\text { integrativa. }\end{array}$ & 2019 \\
\hline 19 & $\begin{array}{l}\text { Paula VAA, Souza ID, Almeida RLM, Santos } \\
\text { KB. }\end{array}$ & $\begin{array}{l}\text { Caracterizar o perfil da formação e atualiza- } \\
\text { ção dos enfermeiros assistenciais e avaliar o } \\
\text { conhecimento sobre o tratamento de feridas } \\
\text { em um hospital público de ensino da Zona da } \\
\text { Mata Mineira. }\end{array}$ & IV & $\begin{array}{l}\text { Estudo } \\
\text { observacional, } \\
\text { descritivo e } \\
\text { quantitativo. }\end{array}$ & 2019 \\
\hline 20 & $\begin{array}{c}\text { Mendes DC, Santos BCOS, Oliveira LB, } \\
\text { Sabino LF. }\end{array}$ & $\begin{array}{c}\text { Mostrar a importância da nutrição como fator de } \\
\text { interferência no processo de cicatrização, expondo } \\
\text { sua relevância em cada etapa. }\end{array}$ & V & $\begin{array}{l}\text { Revisão da } \\
\text { literatura. }\end{array}$ & 2018 \\
\hline 21 & $\begin{array}{c}\text { Moraes JT, BorgesEL,Lisboa CR, Cordeiro } \\
\text { DCO, Rosa EG, Rocha NA. }\end{array}$ & $\begin{array}{c}\text { Apresentar na língua portuguesa a terminologia, } \\
\text { conceito e descrição dos estágios da lesão por } \\
\text { pressão estabelecidos pelo NationalPressureUI- } \\
\text { cerAdvisoryPanel em } 2016 .\end{array}$ & IV & $\begin{array}{l}\text { Descritivo, } \\
\text { reflexivo do } \\
\text { tipo ensaio } \\
\text { teórico. }\end{array}$ & 2016 \\
\hline 22 & $\begin{array}{l}\text { SilvaSAO,Martins FA, Silva AS, GhelenMH,- } \\
\text { Diaz CMG, Martins ESR. }\end{array}$ & $\begin{array}{l}\text { Avaliar, por meio da literatura, o conhecimento } \\
\text { do enfermeiro para diagnosticar o biofilme em } \\
\text { feridas. }\end{array}$ & IV & $\begin{array}{l}\text { Pesquisa } \\
\text { narrativa. }\end{array}$ & 2018 \\
\hline
\end{tabular}


DISCUSSÃO

Lage $^{4}$ aponta que a avaliação constante da pele, cuidados de higiene adequados e a gestão da continência são atividades fundamentais da equipe de enfermagem na prevenção e detecção na DAI. A observação periódica da pele e os cuidados preventivos devem ser especialmente direcionados para pessoas que apresentam algum tipo de incontinência correlacionada a comorbidades, massa corporal e mobilidade passiva.

Segundo Tavares ${ }^{2}$ a proteção da pele é uma etapa essencial, sendo por isso aconselhado a utilização de a creme barreira de longa duração ou de película polimérica em spray. Os protetores de pele com petrolatos e a base de óxido de zinco são utilizados como protetores da pele contra as irritações e hidratação, pela sua fácil acessibilidade e custo reduzido porém se faz importante compreender o uso de determinadas terapias tópicas, pois cremes e pomadas não apresentam um efeito barreira eficaz, a sua coloração branca e opaca impede uma observação adequada do estado da pele, permanecendo uma fina camada que pode provocar lesões cutâneas na tentativa de remoção.

Lage $^{4}$ identifica que o risco maior de incontinência urinária está no sexo feminino e este fatordeve-se pelo fato de diferenças anatômicas como comprimento uretral feminino que se apresenta maior nos homens; a anatomia do assoalho pélvico; além da diminuição da pressão de fechamento uretral, associados à hipermobilidade do colo vesical e ao enfraquecimento na musculatura do assoalho pélvico, devido aos efeitos da gestação e do parto no mecanismo de continência, além das alterações hormonais decorrentes da menopausa.

No artigo de Filho ${ }^{10}$ e Lage $^{4}$, estes assumem em simultâneo que o risco da DAl pode aumentar o risco de lesão por pressão, uma vez que a debilidade da pele aumenta a suscetibilidade à fricção e à pressão. Afirmam a importância do enfermeiro saber distinguir a lesão por pressão da DAI, que por exemplo, que as LPP cos-
O Gestor de Feridas (GF), este enfermeiro que atua autonomicamente da área de tratamento de feridas tem compreensão e conhecimento técnico e científico e não pode se se limitar apenas à tomada de decisão clínica mas também deve discutir em equipe as questões éticas de forma lógica e razoóvel, aplicar os princípios éticos na prática e analisar os dilemas éticos com que se depara. tumam apresentar-se como uma lesão única, circular e simétrica sobre uma proeminência óssea e com bordos bem definidos e as lesões por humidade podem ter uma forma irregular, com bordos mal definidos, surgindo frequentemente no tecido adiposo das nádegas, períneo, parte interna das coxas, escroto e vulva.

Strehlow ${ }^{11}$ enfatiza que a SAE é um instrumento que organiza o trabalho a partir da aproximação entre enfermeiro e cliente, identificando necessidades de cuidados e promovendo a qualidade na recuperação da saúde.A SAE direciona a uma sequência da consulta de enfermagem, diagnósticos e intervenções de enfermagem e desta forma estabelecendo critérios importantes para obtenção de resultados positivos.

Ramos $^{1}$ ressalta que a teoria do autocuidado são os conteúdos necessários para atender às exigências específicas como: assegurar atendimento da equipe multiprofissional; assegurar as ações de reabilitação diagnóstica e terapêutica conforme estabelecidas e protocoladas; readaptação no estilo de vida em decorrência das situações propiciadas pelo evento.

Saud $^{12}$ BT e a PR são tratamentos para o câncer de próstata e podem gerar efeitos adversos na função sexual, intestinal e urinaria e que a incontinência urinaria é uma das principais consequências para os homens que são submetidos a estes tratamentos, gerando piora na qualidade de vida do paciente.

O Gestor de Feridas (GF), este enfermeiro que atua autonomicamente da área de tratamento de feridas tem compreensão e conhecimento técnico e científico e não pode se se limitar apenas à tomada de decisão clínica mas também deve discutir em equipe as questões éticas de forma lógica e razoável, aplicar os princípios éticos na prática e analisar os dilemas éticos com que se depara. Segundo Silva ${ }^{13}$ explica queo limite da autonomia do GF não ficou definido, no entanto foi referido que é relevante fornecer as orientações claras em relação aos limites profissionais, que muitas vezes seguem as recomendações ou legislação nacional/ local. À semelhança de qualquer 
enfermeiro que atua em empresas privadas ou pública, o GF age de forma autónoma.

Paula ${ }^{14}$ constata que uma boa prática assistencial, os protocolos assistenciais, comissões de curativos, estabelecer educação continuada, fazendo relevância em especial o protocolos sobre cuidados com lesões cutâneas ou feridas, se tornam uma excelente estratégia de cuidado, otimizando os recursos disponíveis na instituição elevando a qualidade da abordagem dos profissionais evitando divergências entre condutas.

\section{CONCLUSÃO}

As intervenções de enfermagem dirigidas à pessoa com DAl posicionam-se ao nível da prevenção, diagnóstico e cicatrização. De uma forma geral, todos os

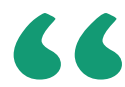

\section{diagnóstico de DAI.}

Na cicatrização está aconselhada a realização de 2 ou 3 etapas: limpar a pele - proteção cutânea - gestão de exsudado. estudos apontaram para a importância de uma adequada inspeção da pele, constituindo-se como o "gold standard" que quer dizer "Padrão ouro" da prevenção e

No que concerne à prevenção da DAI, genericamente encontram-se recomendados 3 etapas no âmbito da intervenção de enfermagem: limpar a pele - aplicação de emolientes/hidratantes proteção cutânea. Na cicatrização está aconselhada a realização de 2 ou 3 etapas: limpar a pele - proteção cutânea gestão de exsudado (se DAl severa).

Portanto, conclui-se este trabalho ressaltando e fazendo ressaltando a importância do gerenciamento do enfermeiro para melhoria do serviço de enfermagem e sua continuidade, fortalecendo o processo do cuidado.

\section{Referências}

1. RamosAF, Pinto MCPF. Dermatite Associada à Incontinência em pacientes portadores de Doenças Crônicas. Revista Uningá, [Internet]. 2016; [cited 2020 Aug 10]. 47(2): 63-69.Availablefrom:http://revista.uninga.br/index. php/uninga/article/view/1265/887

2. Tavares AP, Ramos AF, Vieira EV, Mendonça S, Fonseca C, Lopes M et al. Resultados da intervenção de enfermagem na prevenção e cicatrização da Dermatite Associada à Incontinência (DAl): Revisão sistemática da literatura. Revista Ibero-Americana de Saúde e Envelhecimento, [Internet]. 2016; [cited 2020 Aug 10]. 2(3): 709-722.Availablefrom:http://revistas.uevora.pt/index. php/saude_envelhecimento/article/view/151/252

3. Alcoforado CLGC. Efeito da pomada de óxido de zinco e da película de barreira não irritante na prevenção da dermatite associada à incontinência em idosos hospitalizados: ensaio clínico randomizado [Tese onthe Internet]. Belo Horizonte: Universidade Federal de Minas Gerais; 2018 [cited 2020 Aug 10]. 121 p. Availablefrom: https://repositorio.ufmg.br/bitstream/1843/ ENFC-B3VJHH/1/carla_lucia_goulart_constant_alcoforado.pdf Doutorado em Enfermagem.

4. Lage MA, Silva TBC, Evangelista DR, Rezendo FAC, Netto LSS, Osorio NB et al. Prevalência da incontinência urinária e fecal nos idosos matriculados na Universidade da Maturidade (UMA), no município e Palmas-TO. Humanidades \& Inovação, [Internet]. 2019; [cited 2020 Aug 10]. 6(11): 128-137. Availablefrom: https://revista.unitins.br/index.php/humanidadeseinovacao/ article/view/1579.

5. Greco APC, Guimarães AF, Nascimento TS. Uso do equipamento para controle da incontinência anal no paciente com dermatite: relato de caso. Estima-BrazilianJournalofEnterostomalTherapy, [Internet].2017; [cited 2020 Aug 10] 15(3). Availablefrom: https://www.revistaestima.com.br/index.php/ estima/article/view/549.

6. Soares CB, Hoga LAK, Peduzzi M, Sangaleti C, Yonekura T, Silva DRAD. Integrative Review: ConceptsandMethodsUsedin Nursing. Rev. esc. enferm. USP [Internet]. 2014 Apr [cited 2020 Aug 10]; 48(2): 335-345. Availablefrom: http://www.scielo.br/scielo.php?script=sci_arttext\&pi$\mathrm{d}=$ =S0080-62342014000200335\&lng=en. http://dx.doi.org/10.1590/S00806234201400002000020 .

7. Santos MARC, Galvão MGA. A elaboração da pergunta adequada de pes- quisa. ResidPediatr., Rio de Janeiro,2014; 4(2): 53-56.

8. Galvão TF,PansaniTSA,Harrad D. Principais itens para relatar Revisões sistemáticas e Meta-análises: A recomendação PRISMA. Epidemiol. Serv. de Saúde, [Internet].Brasilia,2015; [cited 2020 Aug 10]; 24(2): 335-342. Tradução para o idioma português do documento: Moher $D$, Liberati $A$, Tetzlaff J, Altman DG, The PRISMA Group. PreferredReportingltems for Systematic Reviews and Meta-Analyses: The PRISMA Statement. Availablefrom: www. prisma-statement.org.

9. Agency For Health Care ReseaRch And Quality (Rockville) (Org.). Quality Improvement and monitoring at your fingertips. 2016; [cited 2020 Aug 10]; Availablefrom: www.qualityindicators.ahrq.gov

10. Filho JSR; De Carvalho CGN. Dermatite das fraldas, fisiopatologia e tratamento: revisão de literatura. Revista de Medicina, [Internet].2017; [cited 2020 Aug 10];96(3): 183-186, 2017. Availablefrom: https://www.revistas. usp.br/revistadc/article/view.

11. Strehlow BR, Fortes VLF, Amarante MV. Dermatite Associada à Incontinência em Idosos Hospitalizados: Conhecimento Autorreferido de Enfermeiros. Revista de Pesquisa: Cuidado é Fundamental Online, [Internet].2018; [cited 2020 Aug 10];10(3): 801-809.Availablefrom: http://www.seer.unirio. br/index.php/cuidadofundamental/article/view/6207/pdf_1

12. Saud LF, Campos GV, Maniezzo R, Marques YNP, Rezendo LF. A incidência de Incontinência Urinária nos tratamentos de Braquiterapia e Prostatectomia radical no câncer de próstata: Abordagem de abrangência em prol da prática clínica. Revista da Universidade Vale do Rio Verde, [Internet].2018[cited 2020 Aug 10];16(1). Availablefrom: http://periodicos.unincor.br/index. php/revistaunincor/article/view/3730.

13. Silva SÃO, Martins FS, Silva AS, Ghelen MH, Dias CMG, Martins ESR. 0 enfermeiro no diagnóstico e tratamento de biofilme em feridas. DisciplinarumScientia| Saúde, [Internet]. 2018[cited 2020 Aug 10];19(2): 281-290. Availablefrom: https://periodicos.ufn.edu.br/index.php/disciplinarumS/article/view.

14. Paula VAA, Souza ID, Almeida RLM, Santos KB. O conhecimento dos enfermeiros assistenciais no tratamento de feridas. HU Revista, [Internet]. 2019; [cited 2020 Aug 10];45(3): 295-303. Availablefrom:https://periodicos. ufjf.br/index.php/hurevista/article/view/28666 